Special Report

For reprint orders, please contact: reprints@futuremedicine.com
Biomarkers

in Medicine

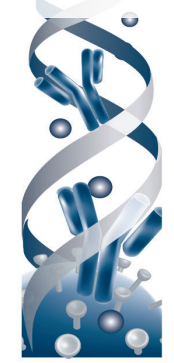

\title{
Potential diagnostic and prognostic biomarkers for cholangiocarcinoma in serum and bile
}

Cholangiocarcinoma (CCA) is a devastating malignancy that is difficult to treat because of its insensitivity to conventional therapies and the inability to detect early tumor formation. Novel molecular techniques have enabled the use of serum and bile markers for CCA diagnosis and prognosis. Herein, we summarize the principal characteristics of serum and bile markers of CCA. Biomarkers such as interleukin-6, matrix metalloproteinases, serotonin (5-hydroxytryptamine) and bile acids have shown promise for improving CCA diagnosis. Several markers such as CYFRA 21-1, MK-1 and C-reactive protein were recently shown to be effective for CCA prognosis.

First draft submitted: 25 December 2015; Accepted for publication: 3 March 2016; Published online: 27 May 2016

Keywords: bile $\bullet$ biomarker $\bullet$ cholangiocarcinoma $\bullet$ serum

Cholangiocarcinoma (CCA) is a devastating malignancy, and the number of cases is currently increasing worldwide [1]. CCA represents $\sim 3 \%$ of all gastrointestinal malignancies, but it is the second most common hepatic malignancy after hepatocellular carcinoma (HCC). The incidence of CCA varies widely in different geographic regions; the highest number of cases occurs in southeast Asia while the lowest number occurs in Australia [2]. CCA is diagnosed based on clinical presentation, ultrasonography, computed tomography, magnetic resonance imaging, endoscopic retrograde cholangiopancreatography, percutaneous transhepatic cholangiography, endoscopic ultrasound, fine-needle aspiration, positron emission tomography and tumor markers. Although there have been advances in the diagnosis and management of CCA, these cancers remain challenging to treat because of their insensitivity to conventional therapies and diagnosticians' inability to detect early tumor formation. Novel targets for diagnostic and prognostic approaches are urgently needed. Various studies conducted over many years have attempted to identify biomarkers in the serum or bile with adequate CCA diagnostic accuracy. These would also be useful for population screening and for the surveillance of disorders for which individuals may be at risk. Herein, we discuss the current diagnostic and prognostic biomarkers for CCA in the serum and bile.

\section{Biomarkers for diagnosis}

Carbohydrate antigen 19-9 and

carcinoembryonic antigen

In clinical practice, the most widely studied and used tumor marker is carbohydrate antigen 19-9 (CA19-9), which is often elevated in CCA $[3,4]$. In a retrospective study of 208 patients with sclerosing cholangitis including 14 patients with CCA, CA19-9 showed $79 \%$ sensitivity and $98 \%$ specificity in CCA diagnosis, with a cut-off value of $129 \mathrm{U} / \mathrm{ml}$ [5]. However, CA19-9 is nondiagnostic for patients who are antigennegative. In addition, CA19-9 levels have been found to be elevated in other conditions such as pancreatic cancer and gastric cancer. A recent study showed that in CCA,
Bin Wang', Liang Chen ${ }^{1}$ \& Hao-Teng Chang ${ }^{\star, 2,3}$ 'Department of Hepatobiliary Surgery, Affiliated Dongyang Hospital of Wenzhou Medical University, Dongyang, Zhejiang 322100, China

${ }^{2}$ Graduate Institute of Basic Medical Science, College of Medicine, China Medical University, Taichung 40402, Taiwan

${ }^{3}$ Department of Computer Science \& Information Engineering, Asia University, Taichung 41354, Taiwan *Author for correspondence: Tel.: +886422052 121 ext 7721 Fax: +886 422333641 htchang@mail.cmu.edu.tw 
CA19-9 had $52.9 \%$ sensitivity and $94.1 \%$ specificity [6], while another study showed $74 \%$ sensitivity, $82 \%$ specificity and $78 \%$ accuracy of CA19-9 in CCA diagnosis [7]. A systematic review discussed the diagnostic performance of CA19-9 for CCA, in which 1264 CCA patients and 2039 controls were analyzed. The authors found $72 \%$ diagnostic sensitivity, $84 \%$ specificity, 4.93 positive likelihood ratio, 0.35 negative likelihood ratio, 15.10 diagnostic odds ratio and an area under the receiver operating characteristic curve (AUC) of 0.83 [8]. Furthermore, carcinoembryonic antigen (CEA) is a common marker used in clinical practice. Although CEA is often elevated in CCA [3,4], it is mainly used to detect colorectal cancer. Only $\sim 30 \%$ of patients with CCA showed elevated CEA levels [9,10]. Moreover, a study showed that evaluation of CEA level alone was neither sensitive nor specific for CCA diagnosis [11].

\section{Interleukin-6}

IL-6 is an inflammatory cytokine produced by cholangiocytes in the presence of inflammatory stimuli. It is also secreted by CCA cells and participates in their mitogenic signaling and survival $[12,13]$. IL-6 upregulates the potent antiapoptotic $\mathrm{Bcl}-2$ protein, myeloid cell leukemia sequence 1 (Mcl-1) via an AKTdependent mechanism [12]. Mcl-1 is integral to tumor necrosis factor-related apoptosis-inducing ligand (TRAIL) resistance in CCA [14]. Consequently, IL-6 inhibition reduces Mcl-1 expression and enhances TRAIL-mediated apoptosis [9]. IL-6 also acts through a mechanism that is dependent upon the signal transducer and activator of transcription (STAT) to enhance Mcl-1 expression [15]. Suppressor of cytokine signaling 3 (SOCS-3) regulates the IL-6/STAT-3 signaling pathway in a feedback manner, and epigenetic silencing of SOCS-3 facilitates sustained IL-6/STAT-3 signaling, resulting in enhanced Mcl-1 expression [16]. This information suggests several approaches for the treatment of the inflammatory subtypes of CCA. For example, neutralizing antibodies against IL- 6 have been used in human clinical trials, and the results suggest that they may be effective therapeutics for treating CCA $[17,18]$. Another study showed that serum IL-6 level is a promising marker, as $73 \%$ sensitivity and $92 \%$ specificity were determined with a cut-off of $25.8 \mathrm{pg} / \mathrm{ml}$. The positive predictive value and negative predictive value were 83 and $87 \%$, respectively [19]. However, elevated levels of IL- 6 are also observed in HCC and other benign biliary lesions. In vitro studies identified IL- 6 as an autocrine growth factor in CCA cell lines $[13,20]$; moreover, IL- 6 is elevated in the serum of patients with CCA and decreases sharply after resection [21].

\section{Mucins}

Serum mucin (MUC) represents another group of promising biomarkers composed of $O$-glycosylated proteins. Biliary MUC4 and serum MUC5AC are tumorassociated mucins that may be useful in diagnosing CCA. Western blotting was used to detect the biliary MUC4 protein in 27\% of CCA and 29\% of primary sclerosing cholangitis cases, but not in other benign and malignant biliary diseases. Serum MUC5AC was detected in $44 \%$ of CCA patients and was negatively correlated with survival [22]. MUC5AC showed $71 \%$ sensitivity, $90 \%$ specificity, $90 \%$ positive predictive value and $69 \%$ negative predictive value for CCA diagnosis [23]. Additionally, the tumor marker CA-125, also known as MUC16 since 2000, may be elevated in CCA but is not specific to this disease. MUC16 may be increased in other gastrointestinal or gynecological malignancies or in cholangiopathies [24]. Specifically, an antibody against M11, a subtype of MUC16 known as MUC16/M11, recognizes the CA-125 epitope expressed in a mucin-like glycoprotein [25-27]. Increased MUC16/M11 levels are correlated with poor survival in patients with intrahepatic CCA with mass-forming type tissues, and are representative of an independent prognostic factor of poor survival [28].

\section{Matrix metalloproteinases}

Matrix metalloproteinases (MMPs) are zinc-dependent proteases that are required for a number of physiological processes, including tissue modeling and repair, embryonic development and angiogenesis [29,30]. Overexpression of MMPs is thought to play an important role in tumor invasion by excessively breaking down the extracellular matrix [31]. MMP-7 and MMP-9 have been reported to be frequently expressed in the tumor tissues of patients with CCA [32]. A prospective study was conducted to investigate whether serum levels of MMP-7 were diagnostic for CCA. This study showed an AUC of 0.84 (95\% CI: 0.78-0.91), with 75\% sensitivity and $78 \%$ specificity. Additionally, elevated levels of serum MMP7 were generally correlated with early and late stages of CCA. Thus, MMP7 was concluded to be a 'potential good marker' of CCA [33].

\section{Serotonin}

Serotonin (5-hydroxytryptamine [5-HT]) is a wellknown neurotransmitter that modulates neural signaling in a wide range of neuropsychological activities. Since its discovery as a mediator of liver regeneration, serotonin has gained considerable attention in liver physiopathology [34]. In contrast to its beneficial effects in enhancing hepatic growth, serotonin has been shown to be negatively correlated with various liver diseases. Given its role in liver regeneration, it is not unexpected 
that serotonin may act as a promoter of malignancy. In a study investigating the impact of serotonin on CCA [35], the expression of TPH-1, the rate-limiting enzyme of serotonin synthesis, was found to be elevated in both CCA cell lines and tumor biopsies from 48 patients; in contrast, cell lines and patients showed downregulation of the serotonin-degrading enzyme. Accordingly, serotonin secretion was elevated in cell suspensions and in bile samples from patients with CCA, but not in samples from patients with intrahepatic lithiasis. Although the addition of serotonin had only a small proliferative effect on starved CCA cell lines, depletion of the molecule by a tryptophan 5-hydroxylase inhibitor markedly suppressed the proliferation of CCA cells in vitro and in a subcutaneous xenograft model. Taken together, these results suggest that serotonin in CCA is not only an inconsequential byproduct but also is actively produced by cancerous cells to promote their growth.

\section{IGF-1}

IGF-1 and IGF-1R were detected in biopsies from 18 patients with CCA. Three CCA cell lines, including HuH-28, TFK-1 and Mz-ChA-1, also expressed IGF-1 and IGF-1R [36]. The biliary IGF-1 concentration was 15-20-fold higher in extrahepatic CCA patients than in patients with pancreatic cancer or benign biliary disorders [37]. The only drawback of this method was the need for an invasive procedure to evaluate biliary IGF-1 levels. IGF-1 showed $82 \%$ sensitivity and $89 \%$ specificity [38].

\section{Minichromosome maintenance}

The expression levels of minichromosome maintenance (MCM) are increased in various malignant human tumors, including prostate cancer, colorectal cancer and neuroblastoma. Kim et al. examined the transcriptional regulation of MCM7 in CCA development and found that MCM7 transactivation may alter the transcriptional regulation of carcinogenic target genes, leading to CCA [39]. MCM replication proteins, particularly MCM5, showed a sensitivity of $66 \%$ compared with $20 \%$ for brush cytology in the detection of CCA [40]

\section{Serum total sialic acid}

Serum levels of total sialic acid (TSA) have been reported to be higher in cell lines undergoing neoplastic transformations, as well as in CCA [41]. The mean value of serum TSA in 89 CCA patients $(2.75 \pm 0.67 \mathrm{mM})$ was significantly higher than that in 38 benign hepatobiliary disease patients $(2.33 \pm 0.69 \mathrm{mM})$ and 43 healthy controls $(1.89 \pm 0.46 \mathrm{mM})$. The AUCs of CCA versus benign hepatobiliary diseases and CCA versus controls were 0.6699 and 0.8558 , respectively [42]. Kongtawelert et al. also reported that the concentra- tion of serum TSA in 69 CCA patients was significantly higher than that in 59 HCC patients, 37 cirrhosis patients, 61 chronic hepatitis patients and 50 healthy blood donors [43].

\section{Tumor type M2 pyruvate kinase}

Tumor type M2 pyruvate kinase (TuM2-PK) concentrations were also found to be significantly increased in patients with CCA. Moreover, the diagnostic performance of TuM2-PK was higher than that of CA19-9, with $84.2 \%$ sensitivity and $90 \%$ specificity compared with $68.4 \%$ sensitivity and $75 \%$ specificity, respectively [44]. Immunohistochemistry for TuM2-PK was performed in 19 CCA patients, and showed that TuM2-PK expression may enhance tumor cell invasion and promote lymph node metastasis of CCA [45].

TGF- $\beta$

TGF- $\beta$, a secreted homodimeric protein, belongs to a large family of pleiotropic factors that transmit signals via heterotetrameric complexes of type I and type II serine/threonine kinase receptors. Inhibition of TGF- $\beta$ receptor in the liver and skeletal muscle significantly reduced the extent of hepatic fibrosis in a thioacetamide-induced liver fibrosis model [46]. Expression of TGF- $\beta 1$ was observed in specimens from $47.4 \%$ of the CCA cases and was significantly correlated with lymph node metastasis, distant metastasis and tumor recurrence [47]. TGF- $\beta$ expression was detected in pairs of CCA and normal bile duct tissues adjacent to the tumor in 47 specimens. Thirty-six specimens $(76.6 \%)$ expressed higher levels of TGF- $\beta$ in tumor tissues than in normal tissues. Furthermore, TGF- $\beta$ expression was related to clinical stage and lymph node and liver metastases [48].

\section{Bile acids}

The concentration and composition of bile acids in patients with CCA was compared with that in patients with biliary tract stones or no biliary disease, and the levels of total bile acid, lithocholic acid and deoxycholic acid were significantly lower in CCA patients [49]. Bile homeostasis was analyzed using a simple nuclear magnetic resonance approach, and bile from patients with CCA exhibited significant changes in glycine-conjugated and taurine-conjugated bile acids, phospholipids, cholesterol and urea [50]. Profiles from CCA patients were clearly different from those of controls and HCC patients.

\section{Alpha fetoprotein}

Serum alpha fetoprotein (AFP) has been widely used in clinical practice as a marker of HCC. Some sera from CCA patients are positive for AFP. Recently, Li et al. 
evaluated serum AFP, CA19-9, CA125 and CEA for diagnosing CCA. They reported that the level of AFP in the serum of CCA patients was significantly lower than that of HCC patients $(\mathrm{p}<0.01)$. However, the value of AUC did not fulfill the criteria to support AFP as a practical diagnostic biomarker of CCA [51]. Levels of AFP, CEA, CA19-9, CA242, and CA50 were measured in the serum of 45 CCA patients and 76 HCC patients. AFP was a sensitive indicator to distinguish CCA from HCC, but its specificity was low. However, using a combination of AFP and CA2 22 markers, the sensitivity, specificity, and accuracy for diagnosing CCA were 88.9, 89.7 and 78.5\%, respectively [52].

\section{Biomarkers for prognosis}

The probability of the long-term survival of CCA patients depends on early diagnosis and, in cases with localized disease, the feasibility of surgical resection [53,54]. Patients not suitable for surgery generally face rapid disease progression with a survival rate of only a few months [53]; thus, prognostic markers are urgently needed to identify CCA patients with poor prognosis who may benefit from more aggressive surgical strategies [55].

With the exception of the factors discussed above, including mucins, CA19-9, and CEA, few studies have evaluated prognostic markers in patients with CCA [56,57]. Other markers such as CYFRA 21-1, MK-1 and C-reactive protein (CRP) have been reported as prognostic markers in patients with CCA. The 3-year recurrence-free survival rates for patients with high and low concentrations of CYFRA $21-1$ are 25 and $76 \%$, respectively [58]. In addition, the expression of MK-1, a tumor-associated antigen encoded by the GA733-2 gene, is found in $79 \%$ of gallbladder carcinomas. MK-1 expression is observed in $\sim 90 \%$ of well-differentiated tubular adenocarcinomas, but in only $\sim 10 \%$ of those that are poorly differentiated. Multivariate analysis has shown that MK-1 expression is an independent prognostic marker that significantly correlates with increased overall survival [59]. Therefore, MK-1 may be used as a prognostic marker for CCA. Gerhardt et al. found that elevated CRP may be an independent predictor of poor survival. In CCA patients, CRP levels may increase because of the complicated tumor-induced structure and cholangitis development [60]. Generally, increased
CRP levels in malignant disease are an inflammatory response to tumor invasion [61]. A lower inflammatory state is correlated with a better prognosis. Saisho et al. found that CRP $<1.0 \mathrm{mg} / \mathrm{dl}$ is a favorable prognostic factor in patients with biliary tract cancers receiving chemotherapy [62].

\section{Conclusion}

Although a number of new serum and bile biomarkers have been recently proposed for CCA, further confirmation is needed to support their applicability in the clinical setting. Some biomarkers appear to be very promising, including MMPs, serotonin, and bile acids, but these biomarkers are useful for discriminating between benign and malignant biliary conditions only in patients who have undergone bile drainage. As a prognosis biomarker, CRP may be a good candidate. Thus, verification of these biomarkers in serum and bile samples is required, as it is only through such validation that measurements of serum and bile markers can be confirmed to be effective for the surveillance of CCA.

\section{Future perspective}

Some biomarkers are very promising, including metalloproteinase, serotonin and bile acids, in discriminating between benign and malignant biliary conditions. CRP seems to be a good prognosis biomarker in CCA patients. These biomarkers need further significant investigation and confirmation, which could provide an effective surveillance for CCA.

\section{Financial \& competing interests disclosure}

The authors have no relevant affiliations or financial involvement with any organization or entity with a financial interest in or financial conflict with the subject matter or materials discussed in the manuscript. This includes employment, consultancies, honoraria, stock ownership or options, expert testimony, grants or patents received or pending, or royalties

The authors thank Dongyang People's Hospital for support for the fee of English editing from Editage.

\section{Open access}

This work is licensed under the Attribution-NonCommercialNoDerivatives 4.0 Unported License. To view a copy of this license, visit http://creativecommons.org/licenses/by-nc-nd/4.0/

\section{Executive summary}

- We summarize the principal characteristics of potential serum and bile markers for cholangiocarcinoma in this mini review.

- Most of markers for cholangiocarcinoma patients are elevated in serum or bile except bile acids.

- MMPs, serotonin, CRP and bile acids are very promising in discriminating between benign and malignant biliary conditions.

- The level of MMPs could reflect the tumor stage and CRP is a practical prognostic biomarker to reflect therapy effectiveness. 


\section{References}

Papers of special note have been highlighted as: $\bullet$ of interest

1 Patel T. Increasing incidence and mortality of primary intrahepatic cholangiocarcinoma in the United States. Hepatology 33(6), 1353-1357 (2001).

2 Shaib Y, El-Serag HB. The epidemiology of cholangiocarcinoma. Semin. Liver Dis. 24(2), 115-125 (2004).

3 Nakeeb A, Lipsett PA, Lillemoe KD et al. Biliary carcinoembryonic antigen levels are a marker for cholangiocarcinoma. Am. J. Surg. 171(1), 147-152; discussion 152-143 (1996).

4 Patel AH, Harnois DM, Klee GG, Larusso NF, Gores GJ. The utility of CA19-9 in the diagnoses of cholangiocarcinoma in patients without primary sclerosing cholangitis. Am. J. Gastroenterol. 95(1), 204-207 (2000).

5 Levy C, Lymp J, Angulo P, Gores GJ, Larusso N, Lindor KD. The value of serum CA19-9 in predicting cholangiocarcinomas in patients with primary sclerosing cholangitis. Dig. Dis. Sci. 50(9), 1734-1740 (2005).

6 Navaneethan U, Lourdusamy V, Poptic E, Hammel JP, Sanaka MR, Parsi MA. Comparative effectiveness of pyruvate kinase M2 in bile, serum carbohydrate antigen $19-9$, and biliary brushings in diagnosing malignant biliary strictures. Dig. Dis. Sci. 60 (4), 903-909 (2015).

7 Lumachi F, Lo Re G, Tozzoli R et al. Measurement of serum carcinoembryonic antigen, carbohydrate antigen 19-9, cytokeratin-19 fragment and matrix metalloproteinase-7 for detecting cholangiocarcinoma: a preliminary case-control study. Anticancer Res. 34(11), 6663-6667 (2014).

8 Liang B, Zhong L, He Q et al. Diagnostic accuracy of serum CA19-9 in patients with cholangiocarcinoma: a systematic review and meta-analysis. Med. Sci. Monit. 21, 3555-3563 (2015).

9 Yachimski P, Pratt DS. Cholangiocarcinoma: natural history, treatment, and strategies for surveillance in high-risk patients. J. Clin. Gastroenterol. 42(2), 178-190 (2008).

10 Blechacz B, Gores GJ. Cholangiocarcinoma: advances in pathogenesis, diagnosis, and treatment. Hepatology 48 (1), 308-321 (2008).

11 Siqueira E, Schoen RE, Silverman W et al. Detecting cholangiocarcinoma in patients with primary sclerosing cholangitis. Gastrointest. Endosc. 56(1), 40-47 (2002).

12 Kobayashi S, Werneburg NW, Bronk SF, Kaufmann SH, Gores GJ. Interleukin- 6 contributes to Mcl-1 up-regulation and TRAIL resistance via an Akt-signaling pathway in cholangiocarcinoma cells. Gastroenterology 128(7), 2054-2065 (2005).

13 Park J, Tadlock L, Gores GJ, Patel T. Inhibition of interleukin 6-mediated mitogen-activated protein kinase activation attenuates growth of a cholangiocarcinoma cell line. Hepatology 30 (5), 1128-1133 (1999).

14 Taniai M, Grambihler A, Higuchi $\mathrm{H}$ et al. Mcl-1 mediates tumor necrosis factor-related apoptosis-inducing ligand resistance in human cholangiocarcinoma cells. Cancer Res. 64(10), 3517-3524 (2004).

15 Isomoto H, Kobayashi S, Werneburg NW et al. Interleukin 6 upregulates myeloid cell leukemia-1 expression through a STAT3 pathway in cholangiocarcinoma cells. Hepatology 42(6), 1329-1338 (2005).

16 Isomoto H, Mott JL, Kobayashi S et al. Sustained IL-6/ STAT-3 signaling in cholangiocarcinoma cells due to SOCS-3 epigenetic silencing. Gastroenterology 132(1), 384-396 (2007).

17 Genovese MC, Fleischmann R, Furst D et al. Efficacy and safety of olokizumab in patients with rheumatoid arthritis with an inadequate response to TNF inhibitor therapy: outcomes of a randomised Phase IIb study. Ann. Rheum. Dis. 73(9), 1607-1615 (2014).

18 Tanaka Y, Martin Mola E. IL-6 targeting compared with TNF targeting in rheumatoid arthritis: studies of olokizumab, sarilumab and sirukumab. Ann. Rheum. Dis. 73(9), 1595-1597 (2014).

19 Cheon YK, Cho YD, Moon JH et al. Diagnostic utility of interleukin-6 (IL-6) for primary bile duct cancer and changes in serum IL-6 levels following photodynamic therapy. Am. J. Gastroenterol. 102(10), 2164-2170 (2007).

20 Okada K, Shimizu Y, Nambu S, Higuchi K, Watanabe A. Interleukin- 6 functions as an autocrine growth factor in a cholangiocarcinoma cell line. J. Gastroenterol. Hepatol. 9(5), 462-467 (1994).

21 Goydos JS, Brumfield AM, Frezza E, Booth A, Lotze MT, Carty SE. Marked elevation of serum interleukin- 6 in patients with cholangiocarcinoma: validation of utility as a clinical marker. Ann. Surg. 227(3), 398-404 (1998).

22 Matull WR, Andreola F, Loh A et al. MUC4 and MUC5AC are highly specific tumour-associated mucins in biliary tract cancer. Br. J. Cancer 98(10), 1675-1681 (2008).

23 Bamrungphon W, Prempracha N, Bunchu N et al. A new mucin antibody/enzyme-linked lectin-sandwich assay of serum MUC5AC mucin for the diagnosis of cholangiocarcinoma. Cancer Lett. 247(2), 301-308 (2007).

24 Chen CY, Shiesh SC, Tsao HC, Lin XZ. The assessment of biliary CA 125, CA19-9 and CEA in diagnosing cholangiocarcinoma - the influence of sampling time and hepatolithiasis. Hepatogastroenterology 49(45), 616-620 (2002).

25 Yin BW, Lloyd KO. Molecular cloning of the CA125 ovarian cancer antigen: identification as a new mucin, MUC16. J. Biol. Chem. 276(29), 27371-27375 (2001).

26 Hardardottir H, Parmley TH 2nd, Quirk JG Jr, Sanders MM, Miller FC, O'Brien TJ. Distribution of CA 125 in embryonic tissues and adult derivatives of the fetal periderm. Am. J. Obstet. Gynecol. 163(6 Pt 1), 1925-1931 (1990).

27 Kobayashi H, Ohi H, Moniwa N, Shinohara H, Terao T. Characterization of CA 125 antigen identified by monoclonal antibodies that recognize different epitopes. Clin. Biochem. 26(5), 391-397 (1993).

28 Higashi M, Yamada N, Yokoyama S et al. Pathobiological implications of MUC16/CA125 expression in intrahepatic cholangiocarcinoma-mass forming type. Pathobiology 79(2), 101-106 (2012).

29 Mosig RA, Dowling O, Difeo A et al. Loss of MMP-2 disrupts skeletal and craniofacial development and results in decreased bone mineralization, joint erosion and defects in osteoblast and osteoclast growth. Hum. Mol. Genet. 16(9), 1113-1123 (2007). 
30 Vairaktaris E, Serefoglou Z, Yapijakis C et al. High gene expression of matrix metalloproteinase-7 is associated with early stages of oral cancer. Anticancer Res. 27(4b), 2493-2498 (2007).

31 Bao W, Fu HJ, Jia LT et al. HER2-mediated upregulation of MMP-1 is involved in gastric cancer cell invasion. Arch. Biochem. Biophys. 499(1-2), 49-55 (2010).

32 Itatsu K, Zen Y, Yamaguchi J et al. Expression of matrix metalloproteinase 7 is an unfavorable postoperative prognostic factor in cholangiocarcinoma of the perihilar, hilar, and extrahepatic bile ducts. Hum. Pathol. 39(5), 710-719 (2008).

33 Leelawat K, Narong S, Wannaprasert J, Ratanashu-Ek T. Prospective study of MMP7 serum levels in the diagnosis of cholangiocarcinoma. World J. Gastroenterol. 16(37), 4697-4703 (2010).

34 Lesurtel M, Graf R, Aleil B et al. Platelet-derived serotonin mediates liver regeneration. Science 312(5770), 104-107 (2006).

35 Alpini G, Invernizzi P, Gaudio E et al. Serotonin metabolism is dysregulated in cholangiocarcinoma, which has implications for tumor growth. Cancer Res. 68(22), 9184-9193 (2008).

36 Alvaro D, Barbaro B, Franchitto A et al. Estrogens and insulin-like growth factor 1 modulate neoplastic cell growth in human cholangiocarcinoma. Am. J. Pathol. 169(3), 877-888 (2006).

37 Alvaro D, Macarri G, Mancino MG et al. Serum and biliary insulin-like growth factor I and vascular endothelial growth factor in determining the cause of obstructive cholestasis. Ann. Intern. Med. 147(7), 451-459 (2007).

38 Chen CY, Tsai WL, Wu HC, Syu MJ, Wu CC, Shiesh SC. Diagnostic role of biliary pancreatic elastase for cholangiocarcinoma in patients with cholestasis. Clin. Chim. Acta 390 (1-2), 82-89 (2008).

39 Kim DW, Kim JY, Moon JH et al. Transcriptional induction of minichromosome maintenance protein 7 $(\mathrm{Mcm} 7)$ in human cholangiocarcinoma cells treated with Clonorchis sinensis excretory-secretory products. Mol. Biochem. Parasitol. 173(1), 10-16 (2010).

40 Ayaru L, Stoeber K, Webster GJ et al. Diagnosis of pancreaticobiliary malignancy by detection of minichromosome maintenance protein 5 in bile aspirates. Br. J. Cancer 98(9), 1548-1554 (2008).

41 Wongkham S, Bhudhisawasdi V, Chau-In S et al. Clinical significance of serum total sialic acid in cholangiocarcinoma. Clin. Chim. Acta 327(1-2), 139-147 (2003).

42 Wongkham S, Boonla C, Kongkham S, Wongkham C, Bhudhisawasdi V, Sripa B. Serum total sialic acid in cholangiocarcinoma patients: an ROC curve analysis. Clin. Biochem. 34(7), 537-541 (2001).

43 Kongtawelert P, Tangkijvanich P, Ong-Chai S, Poovorawan Y. Role of serum total sialic acid in differentiating cholangiocarcinoma from hepatocellular carcinoma. World J. Gastroenterol. 9(10), 2178-2181 (2003).

44 Li YG, Zhang N. Clinical significance of serum tumour M2-PK and CA19-9 detection in the diagnosis of cholangiocarcinoma. Dig. Liver Dis. 41(8), 605-608 (2009).

45 Suzuki H, Komuta M, Bolog A et al. Relationship between 18-F-fluoro-deoxy-D-glucose uptake and expression of glucose transporter 1 and pyruvate kinase M2 in intrahepatic cholangiocarcinoma. Dig. Liver Dis. 47(7), 590-596 (2015).

46 Marquez-Aguirre A, Sandoval-Rodriguez A, GonzalezCuevas J et al. Adenoviral delivery of dominant-negative transforming growth factor beta type II receptor up-regulates transcriptional repressor SKI-like oncogene, decreases matrix metalloproteinase 2 in hepatic stellate cell and prevents liver fibrosis in rats. J. Gene Med. 11(3), 207-219 (2009).

47 Chen Y, Ma L, He Q, Zhang S, Zhang C, Jia W. TGF-beta1 expression is associated with invasion and metastasis of intrahepatic cholangiocarcinoma. Biol. Res. 48, 26 (2015).

48 Zhang BY, Zhang JY, Zhao K, Wu LQ. [Expression of Smad 4 and transforming growth factor-betal, transforming growth factor-beta receptor II in cholangiocarcinoma tissue and its biological significance]. Zhonghua Wai Ke Za Zhi 43(13), 846-849 (2005).

49 Park JY, Park BK, Ko JS, Bang S, Song SY, Chung JB. Bile acid analysis in biliary tract cancer. Yonsei Med. J. 47(6), 817-825 (2006).

- It is negative correlation between cholangiocarcinoma and bile acids.

50 Nagana Gowda GA, Shanaiah N, Cooper A, Maluccio M, Raftery D. Visualization of bile homeostasis using (1) H-NMR spectroscopy as a route for assessing liver cancer. Lipids 44(1), 27-35 (2009).

51 Li Y, Li DJ, Chen J et al. Application of joint detection of AFP, CA19-9, CA125 and CEA in identification and diagnosis of cholangiocarcinoma. Asian Pac. J. Cancer Prev. 16(8), 3451-3455 (2015).

52 Tao LY, Cai L, He XD, Liu W, Qu Q. Comparison of serum tumor markers for intrahepatic cholangiocarcinoma and hepatocellular carcinoma. Am. Surg. 76(11), 1210-1213 (2010).

53 Krasagakis K, Tholke D, Farthmann B, Eberle J, Mansmann U, Orfanos CE. Elevated plasma levels of transforming growth factor (TGF)-beta1 and TGF-beta2 in patients with disseminated malignant melanoma. Br. J. Cancer 77(9), 1492-1494 (1998).

54 Nakagohri T, Asano T, Kinoshita $\mathrm{H}$ et al. Aggressive surgical resection for hilar-invasive and peripheral intrahepatic cholangiocarcinoma. World J. Surg. 27(3), 289-293 (2003).

55 Gores GJ. Cholangiocarcinoma: current concepts and insights. Hepatology 37(5), 961-969 (2003).

56 Abdel Wahab M, Fathy O, Elghwalby N et al. Resectability and prognostic factors after resection of hilar cholangiocarcinoma. Hepatogastroenterology 53(67), 5-10 (2006).

57 Zhou YM, Yang JM, Li B et al. Clinicopathologic characteristics of intrahepatic cholangiocarcinoma in patients with positive serum a-fetoprotein. World J. Gastroenterol. 14(14), 2251-2254 (2008).

58 Uenishi T, Yamazaki O, Tanaka H et al. Serum cytokeratin 19 fragment (CYFRA21-1) as a prognostic factor in 
intrahepatic cholangiocarcinoma. Ann. Surg. Oncol. 15(2), 583-589 (2008).

59 Ikeda T, Nakayama Y, Hamada Y et al. FU-MK-1 expression in human gallbladder carcinoma: an antigenic prediction marker for a better postsurgical prognosis. Am. J. Clin. Pathol. 132(1), 111-117 (2009).

60 Gerhardt T, Milz S, Schepke M et al. C-reactive protein is a prognostic indicator in patients with perihilar cholangiocarcinoma. World J. Gastroenterol. 12(34), 5495-5500 (2006).
61 Uccello M, Malaguarnera G, Corriere T, Biondi A, Basile F, Malaguarnera M. Risk of hepatocellular carcinoma in workers exposed to chemicals. Hepat. Mon. 12(10 hcc), e5943 (2012).

62 Saisho T, Okusaka T, Ueno H, Morizane C, Okada $\mathrm{S}$. Prognostic factors in patients with advanced biliary tract cancer receiving chemotherapy. Hepatogastroenterology 52(66), 1654-1658 (2005).

- The levels of CRP reflect the therapy effectiveness. 